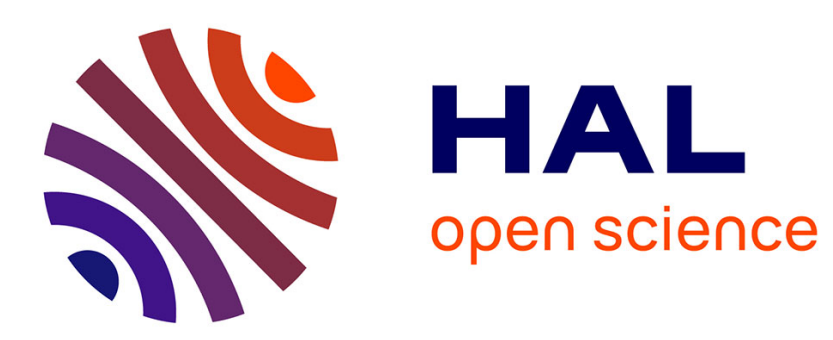

\title{
Théorie des effets de relaxation dans les spectres Mössbauer I. - Notions de base sur la relaxation
}

\author{
F. Hartmann-Boutron
}

\section{To cite this version:}

F. Hartmann-Boutron. Théorie des effets de relaxation dans les spectres Mössbauer I. - Notions de base sur la relaxation. Revue de Physique Appliquée, 1983, 18 (7), pp.413-430. 10.1051/rphysap:01983001807041300 . jpa-00245105

\section{HAL Id: jpa-00245105 https://hal.science/jpa-00245105}

Submitted on 1 Jan 1983

HAL is a multi-disciplinary open access archive for the deposit and dissemination of scientific research documents, whether they are published or not. The documents may come from teaching and research institutions in France or abroad, or from public or private research centers.
L'archive ouverte pluridisciplinaire HAL, est destinée au dépôt et à la diffusion de documents scientifiques de niveau recherche, publiés ou non, émanant des établissements d'enseignement et de recherche français ou étrangers, des laboratoires publics ou privés. 


\title{
Théorie des effets de relaxation dans les spectres Mössbauer I. - Notions de base sur la relaxation
}

\author{
F. Hartmann-Boutron \\ Laboratoire de Spectrométrie Physique (*), U.S.M.G., B.P. 68, 38402 St-Martin d'Hères Cedex, France
}

(Reçu le 23 février 1983, accepté le 24 mars 1983)

\begin{abstract}
Résumé. - Cet article et le suivant sont des versions développées de deux exposés faits à Saclay le 5 mars 1981, lors d'une réunion du Groupe Français de Spectroscopie Mössbauer. Le premier introduit les concepts de base qui interviennent dans les théories de relaxation et le second décrit les différentes méthodes qui permettent de calculer les spectres Mössbauer en présence de relaxation, l'accent étant mis sur les résultats obtenus depuis 1975 (la littérature antérieure à 1975 a'déjà fait l'objet d'une revue par le présent auteur).
\end{abstract}

\begin{abstract}
This paper and the subsequent one are an expanded version of two lectures delivered in Saclay at a meeting of the French Mössbauer Spectroscopy Group (March 5, 1981). The first one is an introduction to the basic concepts of relaxation theory and the second describes the various methods available for computing Mössbauer relaxation spectra, with an emphasis on the results obtained since 1975 (literature prior to 1975 was covered in a previous review paper by this same author).
\end{abstract}

Avant-propos

Depuis bientôt vingt ans, les effets de la relaxation sur les spectres Mössbauer ont fait l'objet de nombreuses études théoriques. Rappelons que les méthodes de calcul utilisées se divisent en deux catégories :

- les méthodes stochastiques, basées sur des modèles phénoménologiques, vante $"$ ).

- les méthodes de perturbation, ou « ab initio », qui présentent deux variantes (« matrice densité » et « résol-

Deux grosses revues ont déjà été consacrées à ces calculs de relaxation :

- la première, parue en 1975 , faisait la synthèse des traitements stochastiques et de type " matrice densité " pour : l'effet Mössbauer, les Corrélations Angulaires Perturbées et l'Orientation Nucléaire (F. HartmannBoutron [1]),

- la seconde, très récente (1981), fait la synthèse des seuls traitements stochastiques pour : les Corrélations Angulaires Perturbées, l'Effet Mössbauer, les expériences sur les Muons et la Résonance Magnétique Nucléaire (S. Dattagupta [2]).

Le formalisme utilisé dans tous ces calculs est malheureusement assez rébarbatif pour le lecteur non spécialiste. La première partie de la présente revue se propose donc d'introduire les notions de base et le vocabulaire (temps de corrélation, densités spectrales, approximation du spectre blanc, approximation séculaire, formalisme de Liouville, supermatrice de relaxation, superopérateur d'évolution, etc.) sur l'exemple le plus simple qu'on puisse trouver, celui d'un spin nucléaire $I=1 / 2$ placé dans un champ statique et soumis à une relaxation par un champ fluctuant classique, ladite relaxation étant détectée par R.M.N. Cet exemple élémentaire devrait faciliter l'abord des revues [1] et [2] et des articles originaux.

La deuxième partie de ce travail est destinée à compléter, en ce qui concerne l'effet Mössbauer, la revue écrite en 1975 par le présent auteur ([1], Chap. VI, p. 310), compte tenu des résultats acquis depuis cette date (notamment par la méthode de la résolvante). Elle est suivie d'une bibliographie récente commentée de façon à rendre plus aisée l'utilisation des articles cités (situation physique à laquelle le traitement s'applique, formes de raie algébriques disponibles).

(*) Laboratoire associé au C.N.R.S. (L.A. 08). 


\section{Relaxation d'un spin $1 / 2$ sous l'effet d'un champ fluctuant.}

Plan

1 Remarque préliminaire.

2 Equation de mouvement propre d'un spin $1 / 2$ dans un champ statique $H_{0}$ parallèle à $\mathrm{O} z$ en l'absence de relaxation.

3 Introduction de la relaxation.

4 Relaxation d'un spin $1 / 2$ en présence d'un champ fluctuant.

5 Formalisme de Liouville.

6 Superopérateurs d'évolution.

7 Application des superopérateurs au calcul de la forme de raie en R.M.N.

8 Opérateurs tensoriels irréductibles.

\section{Remarque préliminaire.}

Le calcul présenté ci-dessous utilise une méthode de perturbation. Ce genre de traitement est délicat car il faut faire des approximations :

- développements en série, solutions par itération

- découplages de moyennes

dont il n'est pas toujours facile de vérifier qu'elles sont justifiées.

Ces approximations ne seront pas discutées en détail dans l'exposé qui suit : nous renvoyons le lecteur intéressé au chapitre VIII du livre d'A. Abragam [3] : The Principles of Nuclear Magnetism.

\section{Equation de mouvement propre d'un spin $1 / 2$ dans un champ magnétique statique $H_{n}$ parallèle à $O z$ en l'absence de relaxation.}

Le spin nucléaire $I=1 / 2$ sera décrit par sa matrice densité $\sigma$.

Rappel : définition de la matrice densité : si le système a une fonction d'onde dépendant du temps $\psi(t)=\sum_{m} C_{m}(t)|m\rangle$, où les $|m\rangle$ sont des vecteurs constants normés à l'unité et les $C_{m}(t)$ satisfont à $\sum_{m}\left|C_{m}(t)\right|^{2}=$ 1 , les éléments de la matrice densité sont définis $\operatorname{par}\langle m|\sigma(t)| n\rangle=C_{m}(t) C_{n}^{*}(t)$.

Pour un spin 1/2, nous caractériserons les états $|m\rangle$ par la valeur propre de $I_{z}, \pm 1 / 2$. La matrice densité a quatre éléments $\left\langle\frac{1}{2}|\sigma| \frac{1}{2}\right\rangle,\left\langle-\frac{1}{2}|\sigma|-\frac{1}{2}\right\rangle,\left\langle\frac{1}{2}|\sigma|-\frac{1}{2}\right\rangle$ et $\left\langle-\frac{1}{2}|\sigma| \frac{1}{2}\right\rangle$. Les deux premiers repèrent les populations $p_{1 / 2}=\left\langle\frac{1}{2}|\sigma| \frac{1}{2}\right\rangle$ et $p_{-1 / 2}=\left\langle-\frac{1}{2}|\sigma|-\frac{1}{2}\right\rangle$ des deux états propres $\left| \pm \frac{1}{2}\right\rangle$ qui satisfont à $p_{1 / 2}+p_{-1 / 2}=1$. Le Hamiltonien du système est :

$$
\mathscr{H}_{\mathrm{o}}=-\hbar \gamma_{\mathrm{n}} H_{\mathrm{n}} I_{\mathrm{z}} \equiv \hbar \omega_{\mathrm{n}} I_{\mathrm{z}},
$$

avec deux valeurs propres $E_{ \pm 1 / 2}= \pm \frac{1}{2} \hbar \omega_{n}$.

L'équation de mouvement de la matrice densité s'écrit :

$$
\frac{\mathrm{d}}{\mathrm{d} t} \sigma=-\frac{i}{\hbar}\left[\mathscr{H}_{0}, \sigma\right]=-i \omega_{\mathrm{n}}\left(I_{\mathrm{z}} \sigma-\sigma I_{\mathrm{z}}\right) .
$$

Soit encore, en insérant dans les produits d'opérateurs la relation de clôture $\left(\sum_{m}|m\rangle\left\langle m|\equiv| \frac{1}{2}\right\rangle\left\langle\frac{1}{2}\right|+\right.$ $\left.\left|-\frac{1}{2}\right\rangle\left\langle-\frac{1}{2}\right|=1\right):$

$$
\frac{\mathrm{d}}{\mathrm{d} t}\langle a|\sigma| b\rangle=-i \omega_{\mathrm{n}} \sum_{m}\left(\left\langle a\left|I_{\mathrm{z}}\right| m\right\rangle\langle m|\sigma| b\rangle-\langle a|\sigma| m\rangle\left\langle m\left|I_{\mathrm{z}}\right| b\right\rangle\right) .
$$


D'où l'on tire :

$$
\left.\begin{array}{l}
\frac{\mathrm{d}}{\mathrm{d} t}\left\langle\frac{1}{2}|\sigma| \frac{1}{2}\right\rangle=0 \\
\frac{\mathrm{d}}{\mathrm{d} t}\left\langle-\frac{1}{2}|\sigma|-\frac{1}{2}\right\rangle=0 \\
\frac{\mathrm{d}}{\mathrm{d} t}\left\langle\frac{1}{2}|\sigma|-\frac{1}{2}\right\rangle=-i \omega_{\mathrm{n}}\left\langle\frac{1}{2}|\sigma|-\frac{1}{2}\right\rangle \\
\frac{\mathrm{d}}{\mathrm{d} t}\left\langle-\frac{1}{2}|\sigma|+\frac{1}{2}\right\rangle=+i \omega_{\mathrm{n}}\left\langle-\frac{1}{2}|\sigma| \frac{1}{2}\right\rangle .
\end{array}\right\}
$$

Ces équations montrent que les pulsations propres qui apparaissent dans l'équation de mouvement de $\sigma$ sous l'effet de $\mathscr{X}_{0}$ sont 0 (pour les éléments diagonaux) et $\mp \omega_{n}$ pour les éléments non diagonaux. Les équations (3) sont des cas particuliers de l'équation générale (valable par rapport aux états propres de $\mathscr{H}_{0}$ ) :

$$
\frac{\mathrm{d}}{\mathrm{d} t}\langle a|\sigma| b\rangle=-i \omega_{a b}\langle a|\sigma| b\rangle=-i\left(\frac{E_{a}-E_{b}}{\hbar}\right)\langle a|\sigma| b\rangle .
$$

Relation entre $\sigma$ et les valeurs moyennes des composantes du spin :

$$
\langle\mathbf{I}\rangle=\operatorname{Trace}(\mathbf{I} \sigma)=\sum_{m n}\langle m|\mathbf{I}| n\rangle\langle n|\sigma| m\rangle
$$

d'où :

$$
\left.\begin{array}{l}
\left\langle I_{z}\right\rangle=\frac{1}{2}\left(p_{1 / 2}-p_{-1 / 2}\right) \\
\left.\left\langle I_{+}\right\rangle=\left\langle-\frac{1}{2}|\sigma| \frac{1}{2}\right\rangle\right) \\
\left\langle I_{-}\right\rangle=\left\langle\frac{1}{2}|\sigma|-\frac{1}{2}\right\rangle,
\end{array}\right\}
$$

soit en se reportant aux équations ci-dessus :

$$
\left.\begin{array}{l}
\frac{\mathrm{d}\left\langle I_{z}\right\rangle}{\mathrm{d} t}=0 \\
\frac{\mathrm{d}\left\langle I_{+}\right\rangle}{\mathrm{d} t}=i \omega_{\mathrm{n}}\left\langle I_{+}\right\rangle \\
\frac{\mathrm{d}\left\langle I_{-}\right\rangle}{\mathrm{d} t}=-i \omega_{\mathrm{n}}\left\langle I_{-}\right\rangle,
\end{array}\right\}
$$

qui décrivent la précession de Larmor du spin nucléaire.

3. Introduction de la relaxation. (Abragam [3], Chap. VIII, p. 276-283; F. Hartmann-Boutron [1], p. 298).

La relaxation du spin nucléaire est due à son couplage au reste du cristal (ou " réseau » ou " bain ») qui joue le rôle d'un thermostat de grande capacité calorifique (c'est-à-dire qu'il est insensible à l'état du spin nucléaire). Ici, nous utiliserons pour simplifier, un modèle phénoménologique consistant à supposer que l'effet du "réseau " sur I est le même que celui d'un champ fluctuant aléatoire.

Le spin I va donc être soumis à la fois au Hamiltonien statique $\mathfrak{H}_{0}$ et au Hamiltonien fluctuant phénoménologique $\mathfrak{H}_{1}(t)$ :

$$
\mathscr{H}_{1}(t)=-\hbar \gamma_{\mathrm{n}} \mathbf{H}_{1}(t) . \mathbf{I},
$$

où $\mathbf{H}_{1}(t)$ est un champ fluctuant aléatoire classique tel que :

$$
\overline{H_{1 x}(t)}=0, \quad \overline{H_{1 y}(t)}=0, \quad \overline{H_{1 z}(t)}=0
$$


mais qui possède des fonctions de corrélation d'ordre 2 non nulles. Pour simplifier, on supposera que parmi toutes les fonctions de corrélation d'ordre 2 qu'on peut construire $\overline{\left(H_{1 x}(0) H_{1 x}(t)\right.}, \overline{H_{1 x}(0) H_{1 z}(t)}$, etc.), seules sont non nulles « par raison de symétrie " les trois fonctions :

$$
\overline{H_{1 z}(t) H_{1 z}(t-\tau)}, \quad \overline{\frac{1}{2} H_{1+}(t) H_{1-}(t-\tau)}, \quad \overline{\frac{1}{2} H_{1-}(t) H_{1+}(t-\tau)},
$$

et que ces fonctions sont invariantes par translation dans le temps (c'est-à-dire indépendantes de $t$ ). Pour le champ aléatoire le plus simple, on admettra de plus qu'elles sont toutes trois égales à $\left\langle H_{1}^{2}\right\rangle \mathrm{e}^{-\tau / \tau_{c}}$ où $\tau_{c}$ est le temps de corrélation du champ fluctuant, qui caractérise la perte de mémoire de l'interaction aléatoire.

En présence de $\mathcal{H}_{0}$ et $\mathfrak{H}_{1}(t)$, l'équation d'évolution de la matrice densité s'écrit :

$$
\frac{\mathrm{d} \sigma}{\mathrm{d} t}=-\frac{i}{\hbar}\left[\mathscr{H}_{0}+\mathscr{H}_{1}(t), \sigma\right] .
$$

Pour obtenir les équations de relaxation à partir de ceci, on commence par passer en « représentation d'interaction » (ou-« représentation intermédiaire ») en posant :

$$
\begin{aligned}
\tilde{\sigma}(t) & =\mathrm{e}^{(i / h) \mathcal{S}_{0} t} \sigma(t) \mathrm{e}^{(-i / h) \mathcal{S}_{0} t} \\
\tilde{\mathscr{K}}_{1}(t) & =\mathrm{e}^{(i / h) \mathcal{S}_{0} t} \mathcal{H}_{1}(t) \mathrm{e}^{(-i / h) \mathcal{S}_{0} t} .
\end{aligned}
$$

Le calcul de $\frac{\mathrm{d}}{\mathrm{d} t} \tilde{\sigma}(t)=\frac{\mathrm{d}}{\mathrm{d} t}\left(\mathrm{e}^{(i / h) x_{0} t} \sigma(t) \mathrm{e}^{(-i / h) x_{0} t}\right)$ montre que l'équation (8) est alors remplacée par :

$$
\frac{\mathrm{d} \tilde{\sigma}}{\mathrm{d} t}=-\frac{i}{\hbar}\left[\tilde{\mathscr{H}}_{1}(t), \tilde{\sigma}(t)\right] \text {. }
$$

L'intérêt de ce changement de représentation (analogue au passage dans le « référentiel tournant » en R.M.N.) est le suivant. $\operatorname{Si} \mathscr{H}_{1}(t)$ et donc $\tilde{\mathscr{H}}_{1}(t)$ étaient nuls, $\tilde{\sigma}(t)$ serait constant. Par suite, si (comme nous allons le supposer maintenant) $\mathcal{H}_{1}(t)$ et $\tilde{H}_{1}(t)$ sont non nuls mais " petits ", la variation de $\tilde{\sigma}(t)$ est lente et l'équation $\left(9^{\prime}\right)$ peut être résolue par itération (méthode de perturbation) :

$$
\tilde{\sigma}(t)=\tilde{\sigma}(0)-\frac{i}{\hbar} \int_{0}^{t}\left[\tilde{\mathscr{Y}}_{1}\left(t^{\prime}\right), \tilde{\sigma}(0)\right] \mathrm{d} t^{\prime}-\frac{1}{\hbar^{2}} \int_{0}^{t} \mathrm{~d} t^{\prime} \int_{0}^{t} \mathrm{~d} t^{\prime \prime}\left[\tilde{\mathscr{H}}_{1}\left(t^{\prime}\right)\left[\tilde{\mathscr{H}}_{1}\left(t^{\prime \prime}\right), \tilde{\sigma}(0)\right]\right]+\cdots
$$

dont la dérivée est

$$
\frac{\mathrm{d} \tilde{\sigma}}{\mathrm{d} t}=-\frac{i}{\hbar}\left[\tilde{\mathscr{H}}_{1}(t), \tilde{\sigma}(0)\right]-\frac{1}{\hbar^{2}} \int_{0}^{t}\left[\tilde{\mathscr{H}}_{1}(t)\left[\tilde{\mathscr{H}}_{1}\left(t^{\prime}\right), \tilde{\sigma}(0)\right]\right] \mathrm{d} t^{\prime}+\cdots
$$

$\overline{\widetilde{\mathfrak{H}}_{1}(t)}$ étant nul, le premier terme du second membre disparaît et il reste :

$$
\frac{\mathrm{d} \tilde{\sigma}}{\mathrm{d} t}=-\frac{1}{\hbar^{2}} \int_{0}^{t} \overline{\left[\tilde{\mathscr{H}}_{1}(t),\left[\tilde{\mathscr{H}}_{1}\left(t^{\prime}\right), \tilde{\sigma}(0)\right]\right] \mathrm{d} t^{\prime}}+\cdots
$$

A ce stade, on fait un certain nombre d'approximations :

- en principe, il faut moyenner sur tout le second membre. La moyenne est restreinte aux produits $\tilde{\mathscr{H}}_{1}(t) \tilde{\mathcal{H}}_{1}\left(t^{\prime}\right)$,

- dans l'intégrale, $\tilde{\sigma}(0)$ est remplacé par $\tilde{\sigma}(t)$,

- après introduction d'une nouvelle variable : $\tau=t-t^{\prime}$, la limite supérieure de l'intégrale est étendue à l'infini.

Compte tenu de l'invariance des fonctions de corrélation par translation dans le temps, l'équation (13) devient :

$$
\left.\frac{\mathrm{d} \tilde{\sigma}}{\mathrm{d} t}=-\frac{1}{\hbar^{2}} \int_{0}^{\infty} \mathrm{d} \tau\left[\overline{\tilde{\mathscr{S}}_{1}(t),\left[\tilde{\mathscr{T}}_{1}(t-\tau)\right.}, \tilde{\sigma}(t)\right]\right] .
$$

C'est l'équation pilote (« master equation ») de la relaxation. 
Pour simplifier encore davantage, on peut admettre que les fonctions de corrélation de $\mathbf{H}$ sont invariantes lorsqu'on change $\tau$ en $-\tau$ (renversement du temps). Ceci permet de remplacer $\int_{0}^{\infty} \operatorname{par} \frac{1}{2} \int_{-\infty}^{+\infty}$ d'où

$$
\left.\frac{\mathrm{d} \tilde{\sigma}}{\mathrm{d} t}=-\frac{1}{2 \hbar^{2}} \int_{-\infty}^{\infty} \mathrm{d} \tau\left[\overline{\left[\tilde{\mathscr{H}}_{1}(t),\left[\tilde{\mathcal{H}}_{1}(t-\tau)\right.\right.}, \tilde{\sigma}(t)\right]\right] .
$$

Cette approximation allège l'exposé et comme Abragam ([3], Chap. VIII), nous l'utiliserons dans ce qui suit; elle revient à négliger de petits déplacements de fréquence associés à la relaxation. Elle n'est pas faite dans les articles les plus récents sur la relaxation Mössbauer.

\section{Relaxation d'un spin $1 / 2$ en présence d'un champ fluctuant.}

4.1 Equations de Relaxation. - Nous avons supposé $\mathfrak{H}_{0}=\hbar \omega_{n} I_{z}$ et :

$$
\mathscr{H}_{1}(t)=-\hbar \gamma_{\mathrm{n}}\left(H_{1 \mathrm{z}}(t) I_{\mathrm{z}}+\frac{1}{2} H_{1-}(t) I_{+}+\frac{1}{2} H_{1+}(t) I_{-}\right) .
$$

En représentation d'interaction :

$$
\frac{\tilde{\mathscr{K}}_{1}(t)}{-\hbar \gamma_{\mathrm{n}}}=\mathrm{e}^{i \omega_{\mathrm{n}} t I_{z}}\left(H_{1 \mathrm{z}}(t) I_{\mathrm{z}}+\frac{1}{2} H_{1-}(t) I_{+}+\frac{1}{2} H_{1+}(t) I_{-}\right) \mathrm{e}^{-i \omega_{\mathrm{n}} t I_{\mathbf{z}}}
$$

$\mathrm{e}^{ \pm i \omega_{\mathrm{n}} I_{\mathrm{z}} t}$ commute avec $I_{\mathrm{z}}$. Donc, le premier terme est égal à $H_{1 \mathrm{z}}(t) I_{\mathrm{z}}$.

D'autre part, compte tenu des règles de sélection de $I_{+}$:

$$
\mathrm{e}^{i \omega_{\mathbf{n}} I_{\mathbf{z}} t} I_{+} \mathrm{e}^{-i \omega_{\mathbf{n}} I_{\mathbf{z}} t}=\mathrm{e}^{i \omega_{\mathbf{n}} t} I_{+} .
$$

Bref :

$$
\tilde{\mathcal{K}}_{1}(t)=H_{1 \mathrm{z}}(t) I_{z}+\frac{1}{2} \mathrm{e}^{i \omega_{\mathrm{n}} t} H_{1-}(t) I_{+}+\frac{1}{2} \mathrm{e}^{-i \omega_{\mathrm{n}} t} H_{1+}(t) I_{-} .
$$

Il faut reporter cette valeur dans l'équation d'évolution de $\tilde{\sigma}$ et calculer toutes les quantités $\frac{\mathrm{d}\langle a|\tilde{\sigma}| b\rangle}{\mathrm{d} t}$ en développant le double commutateur du $2^{\mathrm{e}}$ membre :

$$
\begin{aligned}
& \frac{\mathrm{d}}{\mathrm{d} t}\langle a|\tilde{\sigma}| b\rangle=-\frac{1}{2 \hbar^{2}}\langle a| \int_{-\infty}^{\infty} \mathrm{d} \tau\left\{\overline{\tilde{\mathscr{K}}_{1}(t) \tilde{\mathscr{H}}_{1}(t-\tau)} \tilde{\sigma}(t)-\right.
\end{aligned}
$$

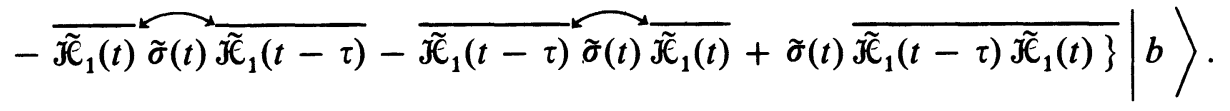

A titre d'exemple, nous allons donner le détail du calcul de la première ligne du double commutateur dans le cas de $-\frac{\mathrm{d}}{\mathrm{d} t}\left\langle\frac{1}{2}|\tilde{\sigma}| \frac{1}{2}\right\rangle$. Avant intégration sur $\tau$, elle s'écrit :

$$
\frac{1}{2 \hbar^{2}}\left\langle\frac{1}{2}\left|\overline{\tilde{\mathscr{H}}_{1}(t) \tilde{\mathscr{H}}_{1}(t-\tau)} \tilde{\sigma}(t)\right| \frac{1}{2}\right\rangle
$$

soit,en insérant deux fois la relation de clôture et en explicitant $\tilde{\mathfrak{H}}_{1}$ :

$$
\begin{aligned}
& =\frac{\gamma_{\mathrm{n}}^{2}}{2} \sum_{\substack{n \\
n}}\left\langle\frac{1}{2}\left|\left\{\frac{1}{2} \mathrm{e}^{i \omega_{\mathrm{n}} t} H_{1-}(t) I_{+}+\frac{1}{2} \mathrm{e}^{-i \omega_{\mathrm{n}} t} H_{1+}(t) I_{-}+H_{12}(t) I_{\mathrm{z}}\right\}\right| n\right\rangle \\
& \times \overline{\left\langle n\left|\left\{\frac{1}{2} \mathrm{e}^{i \omega_{n}(t-\tau)} H_{1_{-}}(t-\tau) I_{+}+\frac{1}{2} \mathrm{e}^{-i \omega_{n}(t-\tau)} H_{1_{+}}(t-\tau) I_{-}+H_{1 z}(t-\tau) I_{z}\right\}\right| m\right\rangle} \\
& \times\left\langle m|\tilde{\sigma}(t)| \frac{1}{2}\right\rangle \text {. }
\end{aligned}
$$


Pour obtenir les contributions non nulles, il faut tenir compte des règles de sélection de $I_{+}, I_{-}, I_{z}$ et du fait que, par hypothèse, seules sont différentes de zéro les fonctions de corrélation d'ordre 2 en $\overline{H_{+} H_{-}}, \overline{H_{-} H_{+}}$et $\overline{H_{z} H_{z}}$ Ceci ne laisse subsister que les termes en $\Delta \triangle$ et $\square \square$ :

$$
\begin{aligned}
& \Delta \Delta \rightarrow \frac{1}{8} \mathrm{e}^{i \omega_{\mathrm{n} \tau} \tau} \gamma_{\mathrm{n}}^{2} \overline{H_{1-}(t) H_{1+}(t-\tau)}\left\langle\frac{1}{2}\left|I_{+}\right|-\frac{1}{2}\right\rangle\left\langle-\frac{1}{2}\left|I_{-}\right|+\frac{1}{2}\right\rangle\left\langle\frac{1}{2}|\tilde{\sigma}(t)| \frac{1}{2}\right\rangle \\
& \square \square \rightarrow \frac{1}{2} \gamma_{\mathrm{n}}^{2} \overline{H_{1 \mathrm{z}}(t) H_{1 \mathrm{z}}(t-\tau)}\left\langle\frac{1}{2}\left|I_{\mathrm{z}}\right| \frac{1}{2}\right\rangle\left\langle\frac{1}{2}\left|I_{\mathrm{z}}\right| \frac{1}{2}\right\rangle\left\langle\frac{1}{2}|\tilde{\sigma(t)}| \frac{1}{2}\right\rangle .
\end{aligned}
$$

Après intégration sur $\tau$ la contribution de la première ligne du double commutateur à $-\frac{\mathrm{d}}{\mathrm{d} t}\left\langle\frac{1}{2}|\sigma| \frac{1}{2}\right\rangle$ s'écrit donc :

$$
\left\{\frac{\gamma_{\mathrm{n}}^{2}}{8} \int_{-\infty}^{\infty} \mathrm{e}^{i \omega_{\mathrm{n} \tau} \tau} \overline{H_{1-}(t) H_{1+}(t-\tau)} \mathrm{d} \tau+\frac{\gamma_{\mathrm{n}}^{2}}{8} \int_{-\infty}^{\infty} \overline{H_{1 \mathrm{z}}(t) H_{1 \mathrm{z}}(t-\tau)} \mathrm{d} \tau\right\}\left\langle\frac{1}{2}|\tilde{\sigma}| \frac{1}{2}\right\rangle .
$$

On trouve de même pour la deuxième ligne :

$$
\begin{aligned}
-\left\{\frac{\gamma_{\mathrm{n}}^{2}}{8} \int_{-\infty}^{\infty} \mathrm{e}^{+i \omega_{\mathrm{n}} \tau} \overline{H_{1-}(t) H_{1+}(t-\tau)} \mathrm{d} \tau\right\}\left\langle-\frac{1}{2}|\tilde{\sigma}|-\frac{1}{2}\right\rangle- \\
-\left\{\frac{\gamma_{\mathrm{n}}^{2}}{8} \int_{-\infty}^{+\infty} \overline{H_{1 \mathrm{z}}(t) H_{1 \mathrm{z}}(t-\tau)} \mathrm{d} \tau\right\}\left\langle\frac{1}{2}|\tilde{\sigma}| \frac{1}{2}\right\rangle,
\end{aligned}
$$

pour la troisième ligne :

$$
\begin{aligned}
-\left\{\frac{\gamma_{\mathrm{n}}^{2}}{8} \int_{-\infty}^{\infty} \mathrm{e}^{-i \omega_{\mathrm{n} \tau} \tau} \overline{H_{1-}(t-\tau) H_{1+}(t)} \mathrm{d} \tau\right\}\left\langle-\frac{1}{2}|\tilde{\sigma}|-\frac{1}{2}\right\rangle- & \\
& -\left\{\frac{\gamma_{\mathrm{n}}^{2}}{8} \int_{-\infty}^{\infty} \overline{H_{1 \mathrm{z}}(t-\tau) H_{1 \mathrm{z}}(t)} \mathrm{d} \tau\right\}\left\langle\frac{1}{2}|\tilde{\sigma}| \frac{1}{2}\right\rangle
\end{aligned}
$$

pour la quatrième ligne :

$$
\left\{+\frac{\gamma_{\mathrm{n}}^{2}}{8} \int_{-\infty}^{\infty} \mathrm{e}^{-i \omega_{\mathrm{n} \tau} \tau} \overline{H_{1-}(t-\tau) H_{1+}(t)} \mathrm{d} \tau+\frac{\gamma_{\mathrm{n}}^{2}}{8} \int_{-\infty}^{\infty} \overline{H_{1 \mathrm{z}}(t-\tau) H_{1 \mathrm{z}}(t)} \mathrm{d} \tau\right\}\left\langle\frac{1}{2}|\tilde{\sigma}| \frac{1}{2}\right\rangle .
$$

Lorsqu'on ajoute ces quatre contributions, les termes en $\overline{H_{\mathbf{z}}(t) H_{\mathbf{z}}(t-\tau)}$ s'éliminent et il vient :

$$
\frac{\mathrm{d}}{\mathrm{d} t}\left\langle\frac{1}{2}|\tilde{\sigma}| \frac{1}{2}\right\rangle \equiv \frac{\mathrm{d}}{\mathrm{d} t} \tilde{\sigma}_{1 / 21 / 2}=-\frac{1}{2 T_{1 \mathrm{n}}} \tilde{\sigma}_{1 / 21 / 2}+\frac{1}{2 T_{1 \mathrm{n}}} \tilde{\sigma}_{-1 / 2-1 / 2},
$$

avec un « temps de relaxation longitudinale $" T_{1 \mathrm{n}}$ tel que :

$$
\begin{aligned}
\frac{1}{T_{1 \mathrm{n}}} & =\frac{\gamma_{\mathrm{n}}^{2}}{4}\left\{\int_{-\infty}^{\infty} \mathrm{e}^{i \omega_{\mathrm{n}} \tau} \overline{H_{1-}(t) H_{1+}(t-\tau)} \mathrm{d} \tau+\int_{-\infty}^{\infty} \mathrm{e}^{-i \omega_{\mathrm{n}} \tau} \overline{H_{1-}(t-\tau) H_{1+}(t)} \mathrm{d} \tau\right\} \\
& =\frac{\gamma_{\mathrm{n}}^{2}}{2} \int_{-\infty}^{+\infty} \mathrm{e}^{i \omega_{\mathrm{n}} \tau} \overline{H_{1-}(t) H_{1+}(t-\tau)} \mathrm{d} \tau .
\end{aligned}
$$

De façon analogue :

$$
\frac{\mathrm{d}}{\mathrm{d} t}\left\langle-\frac{1}{2}|\tilde{\sigma}|-\frac{1}{2}\right\rangle \equiv \frac{\mathrm{d}}{\mathrm{d} t} \tilde{\sigma}_{-1 / 2-1 / 2}=+\frac{1}{2 T_{1 \mathrm{n}}} \tilde{\sigma}_{1 / 21 / 2}-\frac{1}{2 T_{1 \mathrm{n}}} \tilde{\sigma}_{-1 / 2-1 / 2} .
$$

Un calcul du même genre pour les éléments non diagonaux de $\sigma$ conduit à :

$$
\frac{\mathrm{d}}{\mathrm{d} t}\left\langle\frac{1}{2}|\tilde{\sigma}|-\frac{1}{2}\right\rangle=-\frac{1}{T_{2 \mathrm{n}}}\left\langle\frac{1}{2}|\tilde{\sigma}|-\frac{1}{2}\right\rangle
$$




$$
\frac{\mathrm{d}}{\mathrm{d} t}\left\langle-\frac{1}{2}|\tilde{\sigma}|+\frac{1}{2}\right\rangle=-\frac{1}{T_{2 \mathrm{n}}}\left\langle-\frac{1}{2}|\tilde{\sigma}| \frac{1}{2}\right\rangle
$$

avec un « temps de relaxation transversale $» T_{2 n}$ tel que :

$$
\frac{1}{T_{2 \mathrm{n}}}=\frac{1}{2 T_{1 \mathrm{n}}}+\frac{1}{T_{2 \mathrm{n}}^{\prime}}
$$

où $1 / T_{1 \text { n }}$ a été défini ci-dessus (Eq. (28)) et :

$$
\frac{1}{T_{2 \mathrm{n}}^{\prime}}=\frac{\gamma_{\mathrm{n}}^{2}}{2} \int_{-\infty}^{\infty} \mathrm{d} \tau \overline{H_{1 \mathrm{z}}(t) H_{1 \mathrm{z}}(t-\tau)}
$$

Il reste à repasser à la représentation « normale » (non d'interaction) en calculant

$$
\sigma=\mathrm{e}^{(-i / \hbar) r e_{0} t} \tilde{\sigma} \mathrm{e}^{(+i / \hbar) r e_{0} t}
$$

ou encore :

$$
\frac{\mathrm{d} \sigma}{\mathrm{d} t}=-\frac{i}{\hbar}\left[\mathscr{H}_{0}, \sigma\right]+\mathrm{e}^{(-i / \hbar) \mathcal{H}_{0} t} \frac{\mathrm{d} \tilde{\sigma}}{\mathrm{d} t} \mathrm{e}^{(+i / \hbar) \mathcal{S}_{0} t}
$$

Ceci conduit aux équations de relaxation cherchées :

$$
\begin{aligned}
& \frac{\mathrm{d} p_{1 / 2}}{\mathrm{~d} t}=-\frac{1}{2 T_{1 \mathrm{n}}} p_{1 / 2}+\frac{1}{2 T_{1 \mathrm{n}}} p_{-1 / 2} \\
& \frac{\mathrm{d} p_{-1 / 2}}{\mathrm{~d} t}=+\frac{1}{2 T_{1 \mathrm{n}}} p_{1 / 2}-\frac{1}{2 T_{1 \mathrm{n}}} p_{-1 / 2} \\
& \frac{\mathrm{d}}{\mathrm{d} t}\left\langle\frac{1}{2}|\sigma|-\frac{1}{2}\right\rangle=\left(-i \omega_{\mathrm{n}}-\frac{1}{T_{2 \mathrm{n}}}\right)\left\langle\frac{1}{2}|\sigma|-\frac{1}{2}\right\rangle \\
& \frac{\mathrm{d}}{\mathrm{d} t}\left\langle-\frac{1}{2}|\sigma|+\frac{1}{2}\right\rangle=\left(+i \omega_{\mathrm{n}}-\frac{1}{T_{2 \mathrm{n}}}\right)\left\langle-\frac{1}{2}|\sigma|+\frac{1}{2}\right\rangle
\end{aligned}
$$

ou encore

$$
\left.\begin{array}{l}
p_{1 / 2}+p_{-1 / 2}=1 \\
\frac{\mathrm{d}}{\mathrm{d} t}\left\langle I_{\mathrm{z}}\right\rangle=-\frac{1}{T_{1 \mathrm{n}}}\left\langle I_{\mathrm{z}}\right\rangle \\
\frac{\mathrm{d}}{\mathrm{d} t}\left\langle I_{-}\right\rangle=\left(-i \omega_{\mathrm{n}}-\frac{1}{T_{2 \mathrm{n}}}\right)\left\langle I_{-}\right\rangle \\
\frac{\mathrm{d}}{\mathrm{d} t}\left\langle I_{+}\right\rangle=\left(+i \omega_{\mathrm{n}}-\frac{1}{T_{2 \mathrm{n}}}\right)\left\langle I_{+}\right\rangle .
\end{array}\right\}
$$

4.2 Densités SPECTRAles. - Comme le montrent les formules (28), (32), (33), les taux de relaxation $1 / T_{1 \mathrm{n}}$ et $1 / T_{2 n}$ s'expriment en fonction des transformées de Fourier des fonctions de corrélation, qu'on appelle encore densités spectrales.

Posons, en effet :

$$
\begin{gathered}
j_{\mathrm{zz}}(\omega)=\int_{-\infty}^{+\infty} \mathrm{e}^{i \omega \tau} \mathrm{d} \tau \gamma_{\mathrm{n}}^{2} \overline{H_{1 \mathrm{z}}(0) H_{1 \mathrm{z}}(-\tau)} \\
j_{-+}(\omega)=\frac{1}{2} \int_{\infty}^{\infty} \mathrm{e}^{i \omega \tau} \mathrm{d} \tau \gamma_{\mathrm{n}}^{2} \overline{H_{1-}(0) H_{1+}(-\tau)}
\end{gathered}
$$


il vient :

$$
\begin{aligned}
& \frac{1}{T_{1 \mathrm{n}}}=j_{-+}\left(\omega_{\mathrm{n}}\right) \\
& \frac{1}{T_{2 \mathrm{n}}}=\frac{1}{2} j_{-+}\left(\omega_{\mathrm{n}}\right)+\frac{1}{2} j_{\mathrm{zz}}(0) .
\end{aligned}
$$

La pulsation propre d'évolution des populations dans le champ $H_{\mathrm{n}}$ est 0 , celle des éléments non diagonaux est $\pm \omega_{\mathrm{n}}$. On voit que les taux de relaxation $1 / T_{1 \mathrm{n}}, 1 / T_{2 \mathrm{n}}$ sont des combinaisons linéaires de densités spectrales prises aux pulsations propres du système.

Comment calcule-t-on les densités spectrales ?

- ou bien à partir de modèles phénoménologiques simples pour les fonctions de corrélation. C'est ce qu'on fera ici ;

- ou bien à l'aide du « théorème de fluctuation dissipation " qui relie les $j(\omega)$ aux « fonctions de réponse " du réseau (susceptibilités $\chi(\omega)$ ) : cf. [1] (Chap. II, Eqs. II.33, II. 39, II. 55).

4.3 Calcul De $1 / T_{1 \mathrm{n}}$ ET $1 / T_{2 \mathrm{n}}$ Dans Le Cas Du Champ fluctuant Le Plus Simple. - Dans ce cas :

$$
\overline{H_{1 \mathrm{z}}(t) H_{1 \mathrm{z}}(t-\tau)}=\frac{1}{2} \overline{H_{1-}(t) H_{1+}(t-\tau)}=\left\langle H_{1}^{2}\right\rangle \mathrm{e}^{-\left|\tau_{i} \tau_{\mathrm{c}}\right|},
$$

d'où

$$
\begin{gathered}
\frac{1}{T_{1 \mathrm{n}}}=2 \gamma_{\mathrm{n}}^{2}\left\langle H_{1}^{2}\right\rangle \frac{1 / \tau_{\mathrm{c}}}{\omega_{\mathrm{n}}^{2}+1 / \tau_{\mathrm{c}}^{2}} \\
\frac{1}{T_{2 \mathrm{n}}}=\gamma_{\mathrm{n}}^{2}\left\langle H_{1}^{2}\right\rangle \frac{1 / \tau_{\mathrm{c}}}{\omega_{\mathrm{n}}^{2}+1 / \tau_{\mathrm{c}}^{2}}+\gamma_{\mathrm{n}}^{2}\left\langle H_{1}^{2}\right\rangle \tau_{\mathrm{c}} .
\end{gathered}
$$

Ces formules montrent que $1 / T_{1 \mathrm{n}}$ et $1 / T_{2 \mathrm{n}}$ dépendent à la fois du temps de corrélation $\tau_{\mathrm{c}}$ de l'interaction fluctuante et de la pulsation propre $\omega_{\mathrm{n}}$ du spin nucléaire. Ce n'est que lorsque $\tau_{\mathrm{c}}$ est très court, $1 / \tau_{\mathrm{c}} \gg \omega_{\mathrm{n}}$, que $1 / T_{1 \mathrm{n}}$ et $1 / T_{2 \mathrm{n}}$ deviennent indépendants de $\omega_{\mathrm{n}}$. On dit alors que le champ fluctuant satisfait à l'approximation du spectre blanc ("White Noise Approximation » ou WNA). Dans ce cas :

$$
\frac{1}{T_{1 \mathrm{n}}}=\frac{1}{T_{2 \mathrm{n}}}=2 \gamma_{\mathrm{n}}^{2}\left\langle H_{1}^{2}\right\rangle \tau_{\mathrm{c}} .
$$

La signification physique de l'approximation du spectre blanc est la suivante : la largeur en $\omega$ des densités spectrales $j_{-+}(\omega), j_{z z}(\omega)$ est de l'ordre de $1 / \tau_{c}$, tandis que le spin nucléaire n'est sensible qu'aux fluctuations de pulsations $\omega_{\mathrm{n}}$ ou 0 . Si $\omega_{\mathrm{n}} \ll 1 / \tau_{\mathrm{c}}, j_{-+}\left(\omega_{\mathrm{n}}\right) \simeq j_{-+}(0)$ et les taux de relaxation deviennent insensibles à la dépendance en fréquence de $j_{-+}$et $j_{z z}$, comme si le spectre du réseau était «blanc ». Notons à ce propos que lorsque l'approximation du spectre blanc est satisfaite, le calcul de relaxation peut être fait dans n'importe quelle base, alors qu'autrement il faut se placer dans la base des états propres de $\mathfrak{H}_{0}$.

Inversement, lorsque l'approximation du spectre blanc n'est pas valable, on peut utiliser le fait que $1 / T_{1 \mathrm{n}}$, $1 / T_{2 \mathrm{n}}$ dépendent des fréquences propres de $\mathfrak{H e}_{0}$ pour obtenir des renseignements plus détaillés sur $\tau_{\mathrm{c}}$ en faisant (lorsque c'est possible) varier ces fréquences propres (par exemple $\omega_{n}$ dans les équations (43) et (44)).

4. 4 CALCUL DE $1 / T_{1 \mathrm{n}}$ ET $1 / T_{2 \mathrm{n}}$ LORSQUe LA RELAXATION DU SPIN NUCLÉAIRE EST DUE À SON COUPLAGE À UN SPIN PARAMAGNÉTIQUE $\mathbf{S}$ (LUI-MÊME SOUMIS À UNE RELAXATION SPIN RÉSEAU). - Supposons que le couplage hyperfin est isotrope :

$$
\mathscr{H}_{1}=A \mathbf{I} . \mathbf{S}(t)^{\circ}
$$

et que la polarisation du spin paramagnétique est négligeable $(\langle S\rangle \ll S)$. Dans ces conditions $\hbar \gamma_{n} \mathbf{H}_{1}=A \mathbf{S}(t)$ et :

$$
\begin{gathered}
\frac{1}{T_{1 \mathrm{n}}}=\frac{A^{2}}{2 \hbar^{2}} \int_{-\infty}^{\infty} \mathrm{d} \tau \mathrm{e}^{i \omega_{\mathrm{n}} \tau} \overline{S_{-}(0) S_{+}(-\tau)} \\
\frac{1}{T_{2 \mathrm{n}}}=\frac{A^{2}}{4 \hbar^{2}} \int_{-\infty}^{+\infty} \mathrm{d} \tau \mathrm{e}^{i \omega_{\mathrm{n}} \tau} \overline{S_{-}(0) S_{+}(-\tau)}+\frac{A^{2}}{2 \hbar^{2}} \int_{-\infty}^{+\infty} \mathrm{d} \tau \overline{S_{\mathrm{z}}(0) S_{\mathrm{z}}(-\tau)}
\end{gathered}
$$


Si le spin électronique est lui-même soumis à un couplage Zeeman $J_{z}=-\hbar \gamma_{\mathrm{s}} \mathbf{H}_{0} . \mathbf{S} \equiv \hbar \omega_{0} S_{\mathrm{z}}$ et à une relaxation, il va, dans les cas les plus simples obéir à des équations analogues à (37) :

$$
\left.\begin{array}{l}
\frac{\mathrm{d}\left\langle S_{\mathrm{z}}\right\rangle}{\mathrm{d} t}=-\frac{1}{T_{1 \mathrm{~s}}}\left\langle S_{\mathrm{z}}\right\rangle \\
\frac{\mathrm{d}\left\langle S_{-}\right\rangle}{\mathrm{d} t}=\left(-i \omega_{0}-\frac{1}{T_{2 \mathrm{~s}}}\right)\left\langle S_{-}\right\rangle \\
\frac{\mathrm{d}\left\langle S_{+}\right\rangle}{\mathrm{d} t}=\left(+i \omega_{0}-\frac{1}{T_{2 \mathrm{~s}}}\right)\left\langle S_{+}\right\rangle,
\end{array}\right\}
$$

où $T_{1 \mathrm{~s}}$ et $T_{2 \mathrm{~s}}$ sont des temps de relaxation électronique.

Pour calculer $1 / T_{1 \mathrm{n}}$ et $1 / T_{2 n}$, il faut connaître les fonctions de corrélation de $\mathbf{S}$. Nous allons admettre ici que, compte tenu des équations (49), on peut écrire de façon phénoménologique :

$$
\begin{aligned}
\overline{S_{-}(0) S_{+}(-\tau)} & =\left\langle S_{-} S_{+}\right\rangle \mathrm{e}^{-i \omega_{0} \tau-|\tau| / T_{2 s}}=\frac{2 S(S+1)}{3} \mathrm{e}^{-i \omega_{0} \tau-|\tau| / T_{2 s}} \\
\overline{S_{\mathrm{z}}(0) S_{\mathrm{z}}(-\tau)} & =\left\langle S_{\mathrm{z}}^{2}\right\rangle \mathrm{e}^{-|\tau| / T_{1 \mathrm{~s}}}=\frac{S(S+1)}{3} \mathrm{e}^{-|\tau| / T_{1 \mathrm{~s}}}
\end{aligned}
$$

$T_{1 \mathrm{~s}}, T_{2 \mathrm{~s}}$ jouant donc le rôle de temps de corrélation du champ fluctuant $A \mathrm{~S}(t) / \hbar \gamma_{\mathrm{n}}$.

Lorsqu'on reporte (50) dans (47) et (48), il vient :

$$
\begin{aligned}
& \frac{1}{T_{1 \mathrm{n}}}=\frac{2 A^{2} S(S+1)}{3 \hbar^{2}} \frac{1 / T_{2 \mathrm{~s}}}{\left(\omega_{0}-\omega_{\mathrm{n}}\right)^{2}+\left(1 / T_{2 \mathrm{~s}}\right)^{2}} \\
& \frac{1}{T_{2 \mathrm{n}}}=\frac{A^{2} S(S+1)}{3 \hbar^{2}} \frac{1 / T_{2 \mathrm{~s}}}{\left(\omega_{0}-\omega_{\mathrm{n}}\right)^{2}+\left(1 / T_{2 \mathrm{~s}}\right)^{2}}+\frac{A^{2} S(S+1)}{3 \hbar^{2}} T_{1 \mathrm{~s}}
\end{aligned}
$$

Dans chacune de ces expressions, le dénominateur du premier terme fait intervenir $\omega_{0}-\omega_{\mathrm{n}}$ qui représente l'énergie mise en jeu dans un « flip-flop » mutuel des deux spins I et S. La fraction $\frac{1 / T_{2 s}}{\left(\omega_{0}-\omega_{n}\right)^{2}+\left(1 / T_{2 s}\right)^{2}}$ est l'équivalent de la fonction $\delta$ qui intervient dans la règle d'or de Fermi pour la probabilité de transition et qui traduit la condition de conservation de l'énergie. Le deuxième terme de $1 / T_{2 n}$ n'a pas cette forme : en effet, il vient de $A S_{\mathrm{z}}(t) I_{\mathrm{z}}$ qui module la fréquence Zeeman de I mais sans le renverser (terme « adiabatique »), ce qui donne seulement lieu à une disparition de la « cohérence transversale » (c'est-à-dire de $\left\langle I_{+}\right\rangle$et de $\left\langle I_{-}\right\rangle$).

Remarque 1 : le passage rigoureux des équations (47), (48) aux équations (52), (53) se fait à l'aide du théorème de fluctuation dissipation (cf. [1], p. 335, Eqs VII. 53 à VII.56).

Remarque 2 : pour un spin paramagnétique standard, $1 / T_{1 \mathrm{~s}}$ et $1 / T_{2 \mathrm{~s}}$ croissent avec la température $; 1 / T_{1 \mathrm{n}}$ passera donc par un maximum pour $1 / T_{2 \mathrm{~s}} \sim\left|\omega_{0}-\omega_{\mathrm{n}}\right|$. A très haute température $1 / T_{1 \mathrm{~s}}, 1 / T_{2 \mathrm{~s}} \gg\left|\omega_{0}-\omega_{\mathrm{n}}\right|$ et l'approximation du spectre blanc est valable. Dans ce cas, et si l'on suppose de surcroît $T_{1 \mathrm{~s}}=T_{2 \mathrm{~s}}$

$$
\frac{1}{T_{1 \mathrm{n}}}=\frac{1}{T_{2 \mathrm{n}}}=\frac{2 A^{2} S(S+1)}{3 \hbar^{2}} T_{1 \mathrm{~s}}
$$

4.5 APPROXIMATION SÉCULAIRE. - En l'absence d'hypothèse simplificatrice sur les fonctions de corrélation, la solution générale de l'équation pilote (14), en représentation d'interaction et par rapport aux états propres de $\mathscr{H}_{0}$, est donnée par un système d'équations de la forme :

$$
\frac{\mathrm{d}}{\mathrm{d} t} \tilde{\sigma}_{a d}=\sum_{b c} \mathrm{e}^{i\left(\omega_{a d}-\omega_{b c}\right) \mathrm{t}} S_{a d, b c} \tilde{\sigma}_{b c}
$$

qui deviennent en représentation normale :

$$
\frac{\mathrm{d}}{\mathrm{d} t} \sigma_{a d}=-i \omega_{a d} \sigma_{a d}+\sum_{b c} S_{a d, b c} \sigma_{b c}
$$


Exemple : equations (36); les constantes de relaxation $S_{a d, b c}$ de l'équation (55') sont analogues aux $-1 / 2 T_{1 \mathrm{n}}$, $-1 / T_{2 \mathrm{n}}$ des équations (36).

Si ces paramètres de relaxation $S_{a d, b c}$ sont « petits " par rapport aux pulsations propres du système, $\omega_{a d}$, $\omega_{b c}, \ldots$, il est possible de démontrer qu'on commet une erreur négligeable en ne conservant que les couplages de relaxation entre éléments de $\sigma$ oscillant à la même pulsation $\left(\omega_{a d}=\omega_{b c}\right)$ : c'est ce qu'on appelle « l'approximation séculaire ". Elle conduit à des découplages entre les évolutions de certains éléments (par exemple entre éléments diagonaux et nọ diagonaux) et donc à un allègement des équations. Il faut cependant noter que, même si l'approximation séculaire n'est pas valable, des découplages analogues peuvent résulter d'hypothèses sur la symétrie des fonctions de corrélation. C'est le cas pour les équations (36), dont la simplicité tient à ce que seules les trois fonctions de corrélation $\left(7^{\prime}\right)$ ont été supposées non nulles.

\subsection{CONDITIONS DE VALIDITÉ DE LA THÉORIE.}

4.6.1 On s'attend à ce que la matrice densité relaxe vers la matrice de Boltzmann correspondant à l'équilibre thermique. Or, les équations (36) décrivent une relaxation vers la matrice unité. Ceci vient de ce que nous avons représenté le couplage de I au " réseau » par un champ fluctuant classique. Dans ce cas les probabilités de transitions $\frac{1}{2} \rightleftarrows-\frac{1}{2}$ sont égales et les populations à l'équilibre sont égales. Ce traitement n'est donc valable que si $\hbar \omega_{\mathrm{n}} / k_{\mathrm{B}} T \ll 1$.

Quand $\hbar \omega_{\mathrm{n}} / k_{\mathrm{B}} T \gtrsim 1$ il est possible (cf. [1], $\S$ IV.5, p. 302) d'obtenir une relaxation vers la matrice de Boltzmann, à condition de traiter le réseau quantiquement. Dans ce cas le Hamiltonien de relaxation s'écrit :

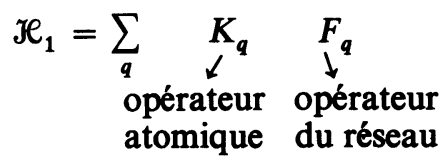

et il faut partir de l'équation de mouvement de la matrice densité du système total (atome + réseau). On prend ensuite la trace par rapport au réseau. Les équations de relaxation obtenues ont le même aspect que précédemment, mais les moyennes sur les fonctions de corrélation du réseau (c'est-à-dire des $F_{q}$ ) font intervenir la matrice densité de Boltzmann du réseau et elles doivent être manipulées avec beaucoup plus de précautions.

4.6.2 Pour obtenir l'équation pilote de la relaxation (14), nous avons :

- résolu l'équation (10) par itération, donc par une méthode de perturbation, ce qui suppose un paramètre de développement petit;

- modifié la moyenne dans l'intégrale du second membre;

- remplacé l'intégrale de 0 à $t$ par une intégrale de 0 à $\infty$;

- remplacé $\sigma(0)$ par $\sigma(t)$ dans l'intégrale.

Ces approximations sont discutées par Abragam ([3], Chap. VIII, p. 282) et dans [4], p. 62. On montre qu'elles sont valables à condition que les temps de relaxation $T_{1 \mathrm{n}}, T_{2 \mathrm{n}}$ soient longs par rapport à $\tau_{\mathrm{c}}$, soit encore :

$$
\frac{1}{T_{1 \mathrm{n}}}, \frac{1}{T_{2 \mathrm{n}}} \ll \frac{1}{\tau_{\mathrm{c}}}
$$

Lorsque l'approximation du spectre blanc est valable $\left(1 / \tau_{c} \gg \omega_{n}\right), 1 / T_{1 n}, 1 / T_{2 n}$ sont de l'ordre de $\frac{\left\langle\mathcal{H}_{1}^{2}\right\rangle}{\hbar^{2}} \tau_{c} ;$ il faut donc :

$$
\frac{\left\langle H_{1}^{2}\right\rangle}{\hbar^{2}} \tau_{\mathrm{c}}^{2} \ll 1
$$

Quand cette condition est satisfaite on peut très bien avoir simultanément :

$$
\frac{1}{T_{1 \mathrm{n}}}, \frac{1}{T_{2 \mathrm{n}}} \sim \frac{\left\langle\mathcal{H}_{1}^{2}\right\rangle}{\hbar^{2}} \tau_{\mathrm{c}} \sim \omega_{\mathrm{n}}
$$

C'est-à-dire que la théorie peut décrire des situations où les effets de relaxation sont comparables aux distances entre raies.

Par contre, lorsque l'approximation du spectre blanc n'est pas valable $\left(1 / \tau_{c} \sim \omega_{n}\right)$, la condition $1 / T_{1 n}$, $1 / T_{2 \mathrm{n}} \ll 1 / \tau_{\mathrm{c}}$ est incompatible avec $1 / T_{1 \mathrm{n}}, 1 / T_{2 \mathrm{n}} \sim \omega_{\mathrm{n}}$. Donc, l'on ne peut décrire que de petits élargissements. 


\section{Formalisme de Liouville ([1], p. 301-307, [5]).}

Dans les équations (55) les éléments de matrice de $\sigma\left(\sigma_{a d}, \sigma_{b c} \ldots\right)$ ont deux indices et les coefficients de relaxation $S_{a d, b c}$ en ont quatre, ce qui suggère de recourir au formalisme de Liouville, dans lequel on utilise des kets à deux indices $|a d\rangle,|b c\rangle$, etc...

Par définition, l'opérateur de Liouville $V^{\times}$correspondant à un opérateur ordinaire $V$ est tel que, quel que soit l'opérateur ordinaire $A$ :

$$
V^{\times} A=[V, A]=V A-A V
$$

soit encore :

$$
\left\langle a\left|V^{\times} A\right| d\right\rangle \equiv\langle a|[V, A]| d\rangle \equiv \sum_{b c}\left\langle a d\left|V^{\times}\right| b c\right\rangle\langle b|A| c\rangle
$$

d'où l'on tire :

$$
\left\langle a d\left|V^{\times}\right| b c\right\rangle=\delta_{d c}\langle a|V| b\rangle-\delta_{a b}\langle c|V| d\rangle .
$$

Dans ce chapitre, nous ne considérerons que le Hamiltonien de Liouville $\mathfrak{H}_{0}^{x}$ correspondant à $\mathfrak{H}_{0}$. Si $|a\rangle$ et $|d\rangle$ sont états propres de $\mathfrak{H}_{0},|a d\rangle$ est état propre de $\mathfrak{H}_{0}^{\times}$avec la valeur propre $\left(E_{a}-E_{d}\right):$ les valeurs propres de $\mathfrak{H}_{0}^{\mathrm{x}} / \hbar$ sont les pulsations propres $\omega_{a d}$ de l'atome, ce qui est physiquement intéressant. Si l'on représente la matrice densité $\sigma$ par une matrice colonne à $(2 I+1)^{2}$ éléments, son équation de mouvement en l'absence de relaxation :

$$
\frac{\mathrm{d} \sigma}{\mathrm{d} t}=-\frac{i}{\hbar}\left[\mathscr{H}_{0}, \sigma\right],
$$

peut s'écrire :

$$
\frac{\mathrm{d} \sigma}{\mathrm{d} t}=-\frac{i}{\hbar} \mathfrak{H}_{0}^{\mathrm{x}} \sigma
$$

De même en présence de relaxation (cf. Eq. (55)) :

$$
\frac{\mathrm{d} \sigma}{\mathrm{d} t}=\left(-\frac{i}{\hbar} \mathcal{H}_{0}^{\times}+S\right) \sigma
$$

où $\left(-\frac{i}{\hbar} \mathfrak{E}_{0}^{\times}+S\right)$ est une « supermatrice " de dimensions $(2 I+1)^{2} \times(2 I+1)^{2}$ dont la partie dissipative $S$ peut être considéree comme une " supermatrice de relaxation "

$\sigma=\left[\begin{array}{l}\sigma_{1 / 21 / 2} \\ \sigma_{-1 / 2-1 / 2} \\ \sigma_{1 / 2-1 / 2} \\ \sigma_{-1 / 21 / 2}\end{array}\right]$

Par exemple pour $I=1 / 2$ et pour $\mathfrak{H}_{0}=\hbar \omega_{\mathrm{n}} I_{2}, \sigma$ est une matrice colonne à 4 éléments (cf. ci-contre),

$$
\begin{gathered}
\left\langle\frac{1}{2} \frac{1}{2}\left|\mathscr{H}_{0}^{\times}\right| \frac{1}{2} \frac{1}{2}\right\rangle=0 \\
\left\langle\frac{1}{2},-\frac{1}{2}\left|\mathcal{H}_{0}^{\times}\right| \frac{1}{2},-\frac{1}{2}\right\rangle=\hbar \omega_{\mathrm{n}}, \quad \text { etc... }
\end{gathered}
$$


et, compte tenu des équations (36), la matrice de $-\frac{i}{\hbar} \mathcal{H}_{0}^{\times}+S$ s'écrit :

\begin{tabular}{|c|c|c|c|c|c|}
\hline & & $\frac{1}{2} \frac{1}{2}$ & $-\frac{1}{2}-\frac{1}{2}$ & $\frac{1}{2}-\frac{1}{2}$ & $-\frac{1}{2} \frac{1}{2}$ \\
\hline$\frac{1}{2}$ & $\frac{1}{2}$ & $-\frac{1}{2 T_{1 \mathrm{n}}}$ & $\frac{1}{2 T_{1 \mathrm{n}}}$ & 0 & 0 \\
\hline$-\frac{1}{2}$ & $-\frac{1}{2}$ & $\frac{1}{2 T_{1 \mathrm{n}}}$ & $-\frac{1}{2 T_{1 \mathrm{n}}}$ & 0 & 0 \\
\hline$\frac{1}{2}$ & $-\frac{1}{2}$ & 0 & 0 & $-i \omega_{n}-\frac{1}{T_{2 n}}$ & 0 \\
\hline$-\frac{1}{2}$ & $\frac{1}{2}$ & 0 & 0 & 0 & $+i \omega_{\mathrm{n}}-\frac{1}{T_{2 \mathrm{n}}}$ \\
\hline
\end{tabular}

A ce stade l'intérêt d'un tel formalisme n'est pas évident. Son avantage n'apparaît que lorsque l'on traite des problèmes plus compliqués, et en relation avec les « superopérateurs d'évolution ».

6. Superopérateurs d'évolution ([1], p. 305, [5], [6]).

6.1 Superopérateur en L'ABSENCe de Relaxation. - Rappel : Lorsque l'on intègre l'équation de Schrödinger correspondant à $\mathcal{H}_{0}$ :

$$
i \hbar \frac{\mathrm{d} \psi}{\mathrm{d} t}=\mathfrak{H}_{0} \psi
$$

il vient :

$$
\psi(t)=\exp \left(-\frac{i}{\hbar} \mathscr{H}_{0} t\right) \psi(0) \equiv U_{0}(t) \psi(0)
$$

où $U_{0}(t)$ est un « opérateur d'évolution » :

$$
U_{0}(t)=\exp \left(-\frac{i}{\hbar} \mathscr{H}_{0} t\right)
$$

De même, si l'on intègre l'équation d'évolution de la matrice densité

$$
\frac{\mathrm{d} \sigma}{\mathrm{d} t}=-\frac{i}{\hbar}\left[\mathscr{H}_{0}, \sigma\right]
$$

il vient :

$$
\sigma(t)=\exp \left(-\frac{i}{\hbar} \mathscr{H}_{0} t\right) \sigma(0) \exp \left(+\frac{i}{\hbar} \mathscr{H}_{0} t\right)=U_{0}(t) \sigma(0) U_{0}^{\dagger}(t) .
$$

Cette intégration peut également se faire dans le formalisme de Liouville. En effet, de l'équation

$$
\frac{\mathrm{d} \sigma}{\mathrm{d} t}=-\frac{i}{\hbar} \mathcal{H}_{0}^{\times} \sigma
$$

il résulte que :

$$
\sigma(t)=\exp \left(\frac{i}{\hbar} \mathcal{H}_{0}^{\times} t\right) \sigma(0) \equiv \mathcal{V}_{0}(t) \sigma(0),
$$

ce qui définit un superopérateur d'évolution $\vartheta_{0}(t)$.

D'après (75) et (73) :

$$
\vartheta_{0}(t) \sigma(0)=U_{0}(t) \sigma(0) U_{0}^{\dagger}(t)
$$


qui s'écrit sous forme matricielle :

$$
\begin{aligned}
\left\langle a\left|\vartheta_{0}(t) \sigma(0)\right| d\right\rangle & \equiv \sum_{b c}\left\langle a d\left|\vartheta_{0}(t)\right| b c\right\rangle\langle b|\sigma(0)| c\rangle \\
& \equiv \sum_{b c}\left\langle a\left|U_{0}(t)\right| b\right\rangle\langle b|\sigma(0)| c\rangle\left\langle c\left|U_{0}^{\dagger}\right| d\right\rangle
\end{aligned}
$$

d'où l'on tire :

$$
\left\langle a d\left|\vartheta_{0}(t)\right| b c\right\rangle=\left\langle a\left|U_{0}(t)\right| b\right\rangle\left\langle c\left|U_{0}^{\dagger}(t)\right| d\right\rangle
$$

ce qui définit les éléments de matrice de $\mho_{0}$ à partir de ceux de $U_{0}$ et $U_{0}^{\dagger}$.

6.2 SuPeropérateur en PRÉSENCE De Relaxation. - Dans ce cas :

$$
\frac{\mathrm{d} \sigma}{\mathrm{d} t}=\left(-\frac{i}{\hbar} \mathfrak{H}_{0}^{\times}+S\right) \sigma
$$

d'où

$$
\sigma(t)=\exp \left[\left(-\frac{i}{\hbar} \mathcal{H}_{0}^{\times}+S\right) t\right] \sigma(0) \equiv \overline{\mathcal{V}}(t) \sigma(0)
$$

ce qui définit un superopérateur d'évolution en présence de relaxation : $\bar{\mho}(t)$ (il n'est, par contre, pas possible de définir $\bar{\mho}(t)$ à partir d'opérateurs d'évolution ordinaires par une équation de type Eq. (76)) (*). La relaxation a pour effet de remplacer, dans l'expression des observables physiques, le superopérateur $\vartheta_{0}(t)$ par le superopérateur $\widehat{v}(t)$.

6.3 CAS DE LA REPRÉSENTATION DE Heisenberg. - Toutes les équations qui précèdent sont écrites en représentation de Schrödinger où la fonction d'onde dépend du temps et les opérateurs n'en dépendent pas.

On peut aussi, comme Martin Blume, travailler en représentation de Heisenberg où les opérateurs dépendent du temps et la fonction d'onde n'en dépend pas.

Dans ce cas, en l'absence de relaxation :

$$
\begin{gathered}
\frac{\mathrm{d} Q}{\mathrm{~d} t}=\frac{i}{\hbar}\left[\mathcal{H}_{0}, Q\right] . \\
Q(t)=\exp \left(\frac{i}{\hbar} \mathcal{H}_{0} t\right) Q(0) \exp \left(-\frac{i}{\hbar} \mathscr{H}_{0} t\right)=U_{0}^{\dagger}(t) Q(0) U_{0}(t) \\
Q(t)=U_{0}(t) Q(0)=\exp \left(+\frac{i}{\hbar} \mathscr{H}_{0}^{\times} t\right) Q(0),
\end{gathered}
$$

ce qui définit un superopérateur d'évolution $\varkappa_{0}(t)$ d'éléments de matrice :

$$
\left\langle a d\left|\mathcal{U}_{0}(t)\right| b c\right\rangle=\left\langle a\left|U_{0}^{\dagger}(t)\right| b\right\rangle\left\langle c\left|U_{0}(t)\right| d\right\rangle .
$$

En présence de relaxation on peut définir de même un superopérateur $\bar{\Psi}(t)$ tel que

$$
Q(t)=\bar{U}(t) Q(0) .
$$

$\overline{\mathcal{W}}(t)$ et $\bar{\vartheta}(t)$ sont liés par les équations générales ([6], Eq. (11))

$$
\left\langle a b|\overline{\mathcal{u}}(t)| a^{\prime} b^{\prime}\right\rangle=\left(\left\langle a^{\prime} b^{\prime}|\overline{\mathcal{V}}(t)| a b\right\rangle\right)^{*}=\left\langle b^{\prime} a^{\prime}|\overline{\mathcal{V}}(t)| b a\right\rangle .
$$

Lorsque la supermattrice de relaxation $S$ est réelle (déplacements de fréquence négligés), il en résulte que :

$$
Q(t) \equiv U(t) Q(0)=\exp \left[\left(+\frac{i}{\hbar} \mathcal{H}_{0}^{\times}+R\right) t\right]
$$

où $R$ est la supermatrice transposée de $S$ ([6], Eq. (12)). Dans le cas traité ici (champ fluctuant classique) : $R=S$, cf. équation (36) ci-dessus.

(*) Cela n'est possible que lorsqu'on quantifie le réseau, cf. [6], équation (8). 
Remarque : en l'absence de relaxation

$$
\begin{aligned}
\sigma(t) & \equiv U_{0}(t) \sigma(0) U_{0}^{+}(t)=U_{0}\left(t-t_{1}\right) U_{0}\left(t_{1}\right) \sigma(0) U_{0}^{+}\left(t_{1}\right) U_{0}^{+}\left(t-t_{1}\right) \\
& =V_{0}\left(t-t_{1}\right) \mho_{0}\left(t_{1}\right) \sigma(0) .
\end{aligned}
$$

Par contre (propriétés des opérateurs hermitiques conjugués)

$$
\begin{aligned}
Q(t) & \equiv U_{0}^{+}(t) Q(0) U_{0}(t)=U_{0}^{+}\left(t_{1}\right) U_{0}^{+}\left(t-t_{1}\right) Q(0) U_{0}\left(t-t_{1}\right) U_{0}\left(t_{1}\right) \\
& \equiv U_{0}\left(t_{1}\right) U_{0}\left(t-t_{1}\right) Q(0) .
\end{aligned}
$$

Il en résulte que les opérateurs $\mho_{0}, \bar{v}$ associés à des temps successifs obéissent à la règle habituelle de multiplication des matrices :

$$
\bar{v}\left(t-t_{0}\right)=\bar{v}\left(t-t_{n}\right) \bar{v}\left(t_{n}-t_{n-1}\right) \ldots \bar{v}\left(t_{1}-t_{0}\right)
$$

mais que (cf. M. Blume) :

$$
\bar{\psi}\left(t-t_{0}\right)=\bar{\psi}\left(t_{1}\right) \ldots \bar{\psi}\left(t_{n}-t_{n-1}\right) \bar{\psi}\left(t-t_{n}\right) .
$$

\section{Application des superopérateurs au calcul de la forme de raie en R.M.N.}

On peut montrer (Abragam [3], Chap. IV, p. 114; [1], p. 293) qu'en l'absence de saturation radiofréquence, la forme de raie d'absorption R.M.N., $I(\omega)$, est proportionnelle à la transformée de Fourier du signal de précession libre $G(t)\left({ }^{1}\right)$ :

$$
\begin{aligned}
I(\omega) & \propto \operatorname{Re} \int_{0}^{\infty} \mathrm{d} t \mathrm{e}^{-i \omega t} G(t) \\
& \propto \operatorname{Re} \int_{0}^{\infty} \mathrm{d} t \mathrm{e}^{-i \omega t} \operatorname{Trace}\left\{I^{-}(0) I^{+}(t)\right\}
\end{aligned}
$$

$I^{+}(t)$ est un opérateur en représentation de Heisenberg. En l'absence de relaxation :

$$
I(\omega) \propto \operatorname{Re} \int_{0}^{\infty} \mathrm{d} t \mathrm{e}^{-i \omega t} \operatorname{Trace}\left\{I^{-} U_{0}^{+}(t) I^{+} U_{0}(t)\right\}
$$

où $U_{0}(t)$ est l'opérateur d'évolution ordinaire défini par l'équation (71); en utilisant l'invariance de la trace par permutation circulaire, l'équation (86) peut encore s'écrire

$$
\begin{aligned}
I(\omega) & \propto \operatorname{Re} \int_{0}^{\infty} \mathrm{d} t \mathrm{e}^{-i \omega t} \operatorname{Trace}\left\{U_{0}(t) I^{-} U_{0}^{+}(t) I^{+}\right\} \\
& \propto \operatorname{Re} \int_{0}^{\infty} \mathrm{d} t \mathrm{e}^{-i \omega t} \sum_{a b c d}\left\langle a\left|U_{0}(t)\right| b\right\rangle\left\langle b\left|I^{-}\right| c\right\rangle\left\langle c\left|U_{0}^{+}(t)\right| d\right\rangle\left\langle d\left|I^{+}\right| a\right\rangle \\
& \propto \operatorname{Re} \int_{0}^{\infty} \mathrm{d} t \mathrm{e}^{-i \omega t} \sum_{a b c d}\left\langle a d\left|V_{0}(t)\right| b c\right\rangle\left\langle b\left|I^{-}\right| c\right\rangle\left\langle d\left|I^{+}\right| a\right\rangle .
\end{aligned}
$$

En présence de relaxation, $\vartheta_{0}(t)$ est remplacé par $\bar{v}(t)$, d'où :

$$
I(\omega) \propto \operatorname{Re} \int_{0}^{\infty} \mathrm{d} t \mathrm{e}^{-i \omega t} \sum_{a b c d}\langle a d|\bar{V}(t)| b c\rangle\left\langle b\left|I^{-}\right| c\right\rangle\left\langle d\left|I^{+}\right| a\right\rangle,
$$

soit pour un spin $I=1 / 2$, compte tenu des règles de sélection de $I^{-}$et $I^{+}$

$$
I(\omega) \propto \operatorname{Re} \int_{0}^{\infty} \mathrm{d} t \mathrm{e}^{-i \omega t}\left\langle-\frac{1}{2} \frac{1}{2}|\bar{\mho}(t)|-\frac{1}{2} \frac{1}{2}\right\rangle\left\langle-\frac{1}{2}\left|I^{-}\right| \frac{1}{2}\right\rangle\left\langle\frac{1}{2}\left|I^{+}\right|-\frac{1}{2}\right\rangle,
$$

( $\left.{ }^{1}\right)$ L'équation (84) contient $\mathrm{e}^{-i \omega t}$ au lieu de $\mathrm{e}^{+i \omega t}$ dans [1], car nous supposons un champ RF tournant $H_{x}=H_{1} \cos \omega t$, $H_{y}=+H_{1} \sin \omega t$, au lieu de $H_{y}=-H_{1} \sin \omega t$ ([1], Eqs. II.45-II.46). 
ou encore, en intégrant formellement :

$$
\begin{aligned}
I(\omega) & \propto \operatorname{Re}\left\langle-\frac{1}{2} \frac{1}{2}\left|\int_{0}^{\infty} \mathrm{d} t \mathrm{e}^{-i \omega t} \exp \left[\left(-\frac{i}{\hbar} H_{0}^{\times}+S\right) t\right]\right|-\frac{1}{2} \frac{1}{2}\right\rangle \\
& \propto \operatorname{Re}\left\langle-\frac{1}{2} \frac{1}{2}\left|\frac{-1}{-i \omega 1-\frac{i}{\hbar} \mathcal{H}_{0}^{x}+S}\right|-\frac{1}{2} \frac{1}{2}\right\rangle
\end{aligned}
$$

On voit que la forme de raie est déterminée par un seul élément de l'inverse de la matrice $i \omega 1-\frac{i}{\hbar} \mathcal{J}_{0}^{\times}+S$. Cette matrice se déduit immédiatement de (68) :

\begin{tabular}{rr|cccc} 
& $\frac{1}{2} \frac{1}{2}$ & $-\frac{1}{2}-\frac{1}{2}$ & $\frac{1}{2}-\frac{1}{2}$ & $-\frac{1}{2} \frac{1}{2}$ \\
\hline$\frac{1}{2}$ & $\frac{1}{2}$ & $-i \omega-\frac{1}{2 T_{1 \mathrm{n}}}$ & $\frac{1}{2 T_{1 \mathrm{n}}}$ & 0 & 0 \\
$-\frac{1}{2}-\frac{1}{2}$ & $\frac{1}{2 T_{1 \mathrm{n}}}$ & $-i \omega-\frac{1}{2 T_{1 \mathrm{n}}}$ & 0 & 0 \\
$\frac{1}{2}-\frac{1}{2}$ & 0 & 0 & $-i \omega-i \omega_{\mathrm{n}}-\frac{1}{T_{2 \mathrm{n}}}$ & 0 \\
$-\frac{1}{2}$ & $\frac{1}{2}$ & 0 & 0 & 0 & $-i \omega+i \omega_{\mathrm{n}}-\frac{1}{T_{2 \mathrm{n}}}$
\end{tabular}

Compte tenu de la factorisation de cette matrice, l'élément de matrice de $\left(-i \omega 1-\frac{i}{\hbar} \mathfrak{X}_{0}^{x}+S\right)^{-1}$ recherché n'est autre que l'inverse du quatrième élément de la diagonale principale, d'où une forme de raie :

$$
I(\omega) \propto \operatorname{Re} \frac{-1}{-i \omega+i \omega_{n}-\frac{1}{T_{2 n}}}=\frac{1 / T_{2 n}}{\left(\omega-\omega_{n}\right)^{2}+\frac{1}{T_{2 n}^{2}}},
$$

qui présente bien un maximum d'absorption à la résonance : $\omega=\omega_{\mathrm{n}}$.

Remarque 1 : Nous avons fait le calcul très progressivement en commençant par exprimer la forme de raie $I(\omega)$ en fonction des opérateurs d'évolution ordinaires $U_{0}$ et $U_{0}^{+}$, puis en introduisant $\mho_{0}(t)$ à partir de la relation (76) entre les éléments de matrice de $\mho_{0}$ et ceux de $U_{0}$ et $U_{0}^{+}$, et finalement en remplaçant $\mho_{0}(t)$ par $\bar{\mho}(t)$.

Cette prudence est nécessaire. En effet, lorsque l'on a un produit formel d'opérateurs de Liouville tels que $\vartheta_{0}(t)$ et d'opérateurs ordinaires tels que $I_{+}, I_{-}$la façon d'écrire ce produit comme un produit d'éléments de matrice n'est pas définie. Ceci vient de ce que les éléments de matrice de $\mathcal{H}_{0}^{\times}$sont obtenus à partir des relations (61), (62) et ceux de $\vartheta_{0}(t)$ à partir de la relation (76). C'est pourquoi toute expression compacte contenant des opérateurs de Liouville et des opérateurs ordinaires devra comporter des crochets indiquant la façon de prendre les éléments de matrice. Par exemple, l'équation (89) peut être représentée par

$$
I(\omega) \propto \operatorname{Re} \int_{0}^{\infty} \mathrm{d} t \mathrm{e}^{i \omega t} \operatorname{Trace}\left[\left\{v_{0}(t) I^{-}\right\} I^{+}\right]
$$

Certains articles contiennent parfois des expressions partiellement dénuées de crochets, ce qui les rend délicates à utiliser.

Remarque 2 : Nous aurions également pu faire le calcul à l'aide des superopérateurs $\mathcal{u}_{0}(t)$ et $\bar{\tau}(t)$. D'après (86) :

$$
\begin{aligned}
I(\omega) & \propto \operatorname{Re} \int_{0}^{\infty} \mathrm{d} t \mathrm{e}^{-i \omega t} \sum_{a b c d}\left\langle a\left|I^{-}\right| b\right\rangle\left\langle b\left|U_{0}^{+}(t)\right| c\right\rangle\left\langle c\left|I^{+}\right| d\right\rangle\left\langle d\left|U_{0}(t)\right| a\right\rangle \\
& \propto \operatorname{Re} \int_{0}^{\infty} \mathrm{d} t \mathrm{e}^{-i \omega t} \sum_{a b c d}\left\langle a\left|I^{-}\right| b\right\rangle\left\langle c\left|I^{+}\right| d\right\rangle\left\langle b a\left|\mathcal{U}_{0}(t)\right| d c\right\rangle,
\end{aligned}
$$


d'où, en remplaçant $\mathcal{u}_{0}$ par $\overline{u_{\text {, }}}$

$$
\begin{gathered}
I(\omega) \propto \operatorname{Re} \int_{0}^{\infty} \mathrm{d} t \mathrm{e}^{-i \omega t}\left\langle-\frac{1}{2}\left|I^{-}\right| \frac{1}{2}\right\rangle\left\langle\frac{1}{2}\left|I^{+}\right|-\frac{1}{2}\right\rangle\left\langle\frac{1}{2}-\frac{1}{2}|\overline{\mathrm{U}}(t)| \frac{1}{2}-\frac{1}{2}\right\rangle \\
I(\omega) \propto \operatorname{Re}\left\langle\frac{1}{2}-\frac{1}{2}\left|\frac{-1}{-i \omega 1+\frac{i}{\hbar} H_{0}^{\mathrm{x}}+R}\right| \frac{1}{2}-\frac{1}{2}\right\rangle,
\end{gathered}
$$

qui conduit bien au même résultat que précédemment (la supermatrice $-i \omega 1+\frac{i}{\hbar} \mathfrak{H}_{0}^{\times}+R$ est ici identique à $-i \omega 1-\frac{i}{\hbar} \mathfrak{C}_{0}^{\times}+S$ au changement près de $\omega_{\mathrm{n}}$ en $-\omega_{\mathrm{n}}$; et l'on prend l'inverse du troisième élément de la diagonale principale, au lieu du quatrième).

8. Opérateurs tensoriels irréductibles. (Edmonds [7], p. 71 ; [1], p. 307).

Dans un certain nombre de cas leur utilisation permet de raccourcir considérablement le calcul du double commutateur de l'équation pilote (14).

On désigne par opérateur tensoriel irréductible $T(k)$ un ensemble de $2 k+1$ opérateurs $T_{k}^{q}(-k \leqslant q \leqslant$ $+k$ ) qui se transforment les uns dans les autres sous une rotation d'angles d'Euler $\alpha \beta \gamma$ de façon analogue aux harmoniques sphériques $Y_{k}^{q}$. Ceci conduit aux deux définitions équivalentes :

$$
D(\alpha \beta \gamma) T_{k}^{q} D^{-1}(\alpha \beta \gamma)=\sum_{q^{\prime}=-k}^{+k} D_{q^{\prime} q}^{k}(\alpha \beta \gamma) T_{k}^{q^{\prime}}
$$

(dans laquelle $D(\alpha, \beta, \gamma)$ est l'opérateur de rotation et la quantité $\mathcal{D}_{q^{\prime} q}^{k}(\alpha \beta \gamma)$ est donnée par l'Eq. 4.1.12 d'Edmonds [7]) ou :

$$
\begin{aligned}
{\left[I_{ \pm}, T_{k}^{q}\right] } & =\sqrt{(k \mp 1)(k \pm q+1)} T_{k}^{q \pm 1} \\
{\left[I_{z}, T_{k}^{q}\right] } & =q T_{k}^{q},
\end{aligned}
$$

avec pour l'hermitique conjugué :

$$
T_{k}^{q \dagger}=(-1)^{q} T_{k}^{-q} .
$$

Les éléments de matrice des $T_{k}^{q}$ obéissent au théorème de Wigner-Eckart ([7], Eq. 5.4.1) qui dit que pour $k$ et $q$ donnés, ils sont tous proportionnels à un même coefficient de Clebsch-Gordan; les propriétés de ce coefficient font qu'à l'intérieur d'une multiplicité I donnée on ne peut définir que des $T_{k}^{q}$ de $k \leqslant 2 I$.

Il est souvent commode d'utiliser des opérateurs normés, c'est-à-dire tels que

$$
\underset{I}{\operatorname{Trace}}\left(T_{k}^{q} T_{k^{\prime}}^{q^{\prime}}\right)=\delta_{k k^{\prime}} \delta_{q q^{\prime}}
$$

Des opérateurs $T_{k}^{q}$ non normés sont fournis par les équivalents opératoriels des harmoniques sphériques (Elliott et Stevens) utilisés en théorie du champ cristallin.

Exemples d'opérateurs tensoriels irréductibles : Opérateurs normés pour $I=1 / 2$ :

$$
\left.\begin{array}{l}
T_{0}^{0}=1 / \sqrt{2} \\
T_{1}^{0}=\sqrt{2} I_{z}, \quad T_{1}^{1}=-I_{+}, \quad T_{1}^{-1}=+I_{-} .
\end{array}\right\}
$$

Opérateurs normés pour $I=1$ :

$$
\left.\begin{array}{l}
T_{0}^{0}=\frac{1}{\sqrt{3}} \\
T_{1}^{0}=\frac{1}{\sqrt{2}} I_{z}, \quad T_{1}^{ \pm 1}=\mp \frac{1}{2} I_{ \pm} \\
T_{2}^{0}=\frac{1}{\sqrt{6}}\left(3 I_{z}^{2}-I(I+1)\right), \quad T_{2}^{ \pm 1}= \pm \frac{1}{2}\left(I_{z} I_{ \pm}+I_{ \pm} I_{z}\right), \quad T_{2}^{ \pm 2}=\frac{1}{2} I_{ \pm}^{2} .
\end{array}\right\}
$$


Application des opérateurs tensoriels aux calculs de relaxation (cf. [1], p. 333) : Pour ceci il faut d'abord développer la matrice densité $\sigma$ en O.T.I. :

$$
\sigma=\sum_{k q} \sigma_{k}^{q^{*}} T_{k}^{q} \equiv \sum_{k q} \sigma_{k}^{q} T_{k}^{q \dagger}
$$

d'où, compte tenu de (105) :

$$
\sigma_{k}^{q}=\operatorname{Trace}\left(\sigma T_{k}^{q}\right)
$$

ainsi, pour $I=1 / 2$ :

$$
\left.\begin{array}{l}
\sigma_{0}^{0}=\frac{1}{\sqrt{2}}\left(p_{1 / 2}+p_{-1 / 2}\right)=\frac{1}{\sqrt{2}} \\
\sigma_{1}^{0}=\frac{1}{\sqrt{2}}\left(p_{1 / 2}-p_{-1 / 2}\right) \\
\sigma_{1}^{1}=-\sigma_{-1 / 2+1 / 2} \\
\sigma_{1}^{-1}=+\sigma_{+1 / 2-1 / 2} .
\end{array}\right\}
$$

On cherche ensuite l'équation d'évolution des $\sigma_{k}^{q}$ en présence de $\mathscr{H}_{0}$ et $\mathscr{H}_{1}(t)$ :

$$
\left.\left.\frac{\mathrm{d}}{\mathrm{d} t}\left(\sum_{k^{\prime} q^{\prime}} \tilde{\sigma}_{k^{\prime}}^{q^{\prime \prime}} T_{k^{\prime}}^{q^{\prime}}\right)=-\frac{1}{2 \hbar^{2}} \int_{-\infty}^{+\infty} \mathrm{d} t \overline{\left[\tilde{\mathscr{H}}_{1}(t),\left[\tilde{\mathscr{H}}_{1}(t-\tau)\right.\right.},\left(\sum_{k^{\prime \prime} q^{\prime \prime}} \tilde{\sigma}_{k^{\prime \prime} q^{\prime \prime}}^{*} T_{k^{\prime \prime}}^{q^{\prime \prime}}\right)\right]\right] .
$$

En multipliant à gauche par $T_{k}^{q \dagger}$ et en prenant la trace sur $I$, il vient :

$$
\left.\left.\frac{\mathrm{d}}{\mathrm{d} t} \tilde{\sigma}_{k}^{q^{*}}=-\frac{1}{2 \hbar^{2}} \int_{-\infty}^{+\infty} \mathrm{d} \tau \underset{I}{\operatorname{Trace}}\left\{T_{k}^{q \dagger} \overline{\left[\tilde{\mathscr{H}}_{1}(t),\left[\tilde{\mathscr{H}}_{1}(t-\tau)\right.\right.},\left(\sum_{k^{\prime \prime} q^{\prime \prime}} \tilde{\sigma}_{k^{\prime \prime} q^{\prime \prime}}^{*} T_{k^{\prime \prime}}^{q^{\prime \prime}}\right)\right]\right]\right\}
$$

Si la relaxation a une symétrie élevée, il arrive que le terme en trace au deuxième membre se simplifie considérablement : ainsi dans l'exemple considéré tout au long de cet article, où $\mathfrak{H}_{1}(t)$ représente le couplage de $I$ à un champ fluctuant isotrope, il apparaît au second membre des quantités telles que :

$$
\underset{I}{\operatorname{Trace}}\left\{T_{k}^{q \dagger}\left[I_{-},\left[I_{+}, T_{k^{\prime}}^{q^{\prime}}\right]\right]\right\}
$$

qui, si l'on se reporte à la définition des $T_{k}^{q}$, est tout simplement égale à :

$$
(k-q)(k+q+1) \delta_{k k^{\prime}} \delta_{q q^{\prime}}
$$

et l'on arrive finalement à l'équation :

$$
\frac{\mathrm{d} \sigma_{k}^{q^{*}}}{\mathrm{~d} t}=\left(i q \omega_{\mathrm{n}}-\mu_{k}^{q}\right) \sigma_{k}^{q^{*}}
$$

avec :

$$
\mu_{k}^{q}=\frac{k(k+1)}{2 T_{1 \mathrm{n}}}+q^{2}\left(\frac{1}{T_{2 \mathrm{n}}}-\frac{1}{T_{1 \mathrm{n}}}\right),
$$

où $T_{1 \mathrm{n}}$ et $T_{2 \mathrm{n}}$ sont donnés par les équations (28), (32), (33).

Pour $I \stackrel{2 \mathrm{n}}{=} 1 / 2, k=1, q=0, \pm 1$ ces équations se réduisent aux équations (36). Mais elles ont l'avantage d'être beaucoup plus générales, puisqu'elles sont valables quels que soient $I, k$ et $q$.

Par exemple, pour $I=1$ elles fournissent à la fois la relaxation de la polarisation $(k=1)$ observée en R.M.N., et celle de l'alignement $(k=2)$ observée en corrélations angulaires perturbées. Ainsi le taux de relaxation de $\left\langle I_{\mathrm{z}}\right\rangle \propto \sigma_{1}^{0}$ est $1 / T_{1 \mathrm{n}}$ et celui de $\left\langle 3 I_{\mathrm{z}}^{2}-I(I+1)\right\rangle \propto \sigma_{2}^{0}$ est $3 / T_{1 \mathrm{n}}$.

Comme le montrent ces exemples, une technique d'observation particulière ne donne souvent accès qu'à une seule composante tensorielle de la matrice densité de l'atome; par ailleurs, lorsque le hamiltonien principal $\mathscr{H}_{0}$ et le hamiltonien de relaxation $\mathscr{H}_{1}(t)$ ont une symétrie élevée, il arrive fréquemment que les équations d'évo- 
lution des différentes composantes tensorielles soient découplées (cf. Eq. (114) ci-dessus). Dans ce cas l'utilisation des opérateurs tensoriels irréductibles permet d'accéder directement à la sous-matrice de $-i \omega \mathbb{1}-\frac{i}{\hbar} \mathfrak{H}_{0}^{\times}+S$ dont on a besoin pour calculer la forme de raie, et évite d'avoir à inverser une énorme matrice. Cette méthode est puissante, mais elle exige la manipulation d'une algèbre spécialisée et assez lourde qui est exposée dans le livre d'Edmonds : Angular Momentum in Quantum Mechanics [7].

\section{Bibliographie}

[1] Hartmann-Boutron, F., Ann. Phys. 9 (1975) 285-356.

[2] Dattagupta, S., Hyp. Int. 11 (1981) 77-126.

[3] Abragam, A., The principles of Nuclear Magnetism (Oxford University Press) 1961.

[4] Hartmann-Boutron, F., J. Physique 40 (1979) 57.

[5] Hartmann-Boutron, F., Spanjaard, D., J. Physique 36 (1975) 307.

[6] Chopin, C., Spanjaard, D., Hartmann-Boutron, F., J. Physique Colloq. 37 (1976) C6-73 (Conférence Mössbauer, Corfou).

[7] Edmonds, A. R., Angular Momentum in Quantum Mechanics (Princeton University Press) 1957. 